MATEC Web of Conferences 28, 04001 (2015)

DOI: $10.1051 /$ matecconf $/ 20152804001$

(C) Owned by the authors, published by EDP Sciences, 2015

\title{
Robot Control System based on Web Application and RFID Technology
}

\author{
Ali Vatankhah Barenji ${ }^{\text {a }}$ and Canberk Değirmenci \\ Department of Mechanical Engineering, Eastern Mediterranean University, Famagusta, North Cyprus Via 10, Mersin, Turkey
}

\begin{abstract}
This paper discusses an integration driven framework for enabling the RFID based identification of parts to perform robotic distributor operations in the random mix based parts control based on web application. The RFID technology senses newly arriving parts to be distribution robot, the robot is able to recognize them and perform cooperative distributing via web-based application. The developed web application control system is implemented in the educational robotic arm. RFID system sends real time information from parts to the web application and web based application makes a decision for control of the robot arm, controller of robot controls the robot as based on the decision from web application. The proposed control system has increases the reconfiguration and scalability of robot system.
\end{abstract}

\section{Introduction}

Faced with the mass customization trends, current and future manufacturing systems are faced to provide the ability of rapid reconfiguration. The objective of reconfiguration is to allow a manufacturing system to change quickly and cost- effectively from its current configuration to another configuration without being taken offline maintaining system effectiveness when sudden changes in customer demands or unpredictable events such as failures and disruptions occur [1]. A manufacturing system, which is made of subsystems that can be complex systems themselves, also one of the important subsystem, is robotic system. Nowadays industrial robots are used in the industry for many purposes such as assembling, packaging, dividing etc., but from changing mass production to mass customization [2] , industrial robots must adapt themselves at an ever-increasing pace to incorporate new information technology and new products as well as new information [3]. This new configuration of adaptation requires reconfiguration for industrial robots. For this work proposed web based reconfigurable control system for distributor industrial robot that consider random arrival of the parts for identification parts and real time decision making, RFID system is used and scheduling of the each part is written on the RFID tags was attached to the part [4].

\section{Literature Review}

In the past few years, with the great improvement of web technology, many approaches have been proposed to implement web-based systems for design and manufacturing. For example, Chui and Wright [5] have developed an Internet-based multimedia educational tool for the design of simple mechanical parts. Reed and Afjeh [6] have used web-based interactive engineering tool for engineering simulation and teaching and learning purposes. Many researchers and practitioners have also made other efforts in web-based engineering applications. there are some researchers that used internet to control their robot; reference [7] suggest to use SOAP protocol and a PC server with ASP.NET framework and also a PC client that has been developed with ASP.NET framework[8]. The suggested protocol takes about 30 seconds to respond due to the overhead in the Web service, since the Web service works as request-response base, it spoils the computation efficiency in the simulator.

Radio Frequency Identification (RFID) is an emerging technology appropriate to a wide range of applications. RFID system consists of three parts namely: RFID reader, RFID antenna and RFID tag. RFID tags offer several broad advantages, including long-distance contact, programmability, generous local storage, and non-line-of-sight scan-ability [9]. In addition, RFID tags' resistance to magnetic interference, water damage, and high temperature make them very suitable for industrial applications [1]. In particular, the use of RFID tags in manufacturing systems enables the collection and processing of real-time manufacturing and product information at all points of the value chain. The aim of this research is to explore and investigate the idea of using RFID and web application for reconfigurable controlling industrial robot for distributor industrial robot that consider random arrival of the parts.

\section{Methodology}

\footnotetext{
${ }^{a}$ Corresponding author: ali.vatankhah.barenji@gmail.com
} 
This paper discusses a method and its application to a robotic distributor cell for performance of distributing operations in a random mix parts. The method is utilizing the RFID-based sensing identification mechanism and discusses the web-based application for real time control robot arm. Figure 1 shows the architecture of RFID and Web based control system for distributor Robot arm. The architecture consists of four modules; namely, RFID system, Web based control application, Embedded Computer, Robot Control System.

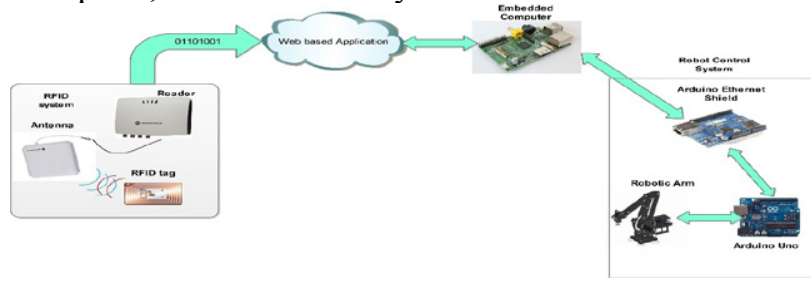

Figure 1. The architecture of RFID and Web based application for real time control

\section{RFID System}

It is responsible for identifying parts and sending related data to the web application. According tag data related data, consists of scheduling information for a part; this data is written on the tag, as based on the process planning.

\section{Web based Application}

The architecture of web application is illustrated in the Figure 2

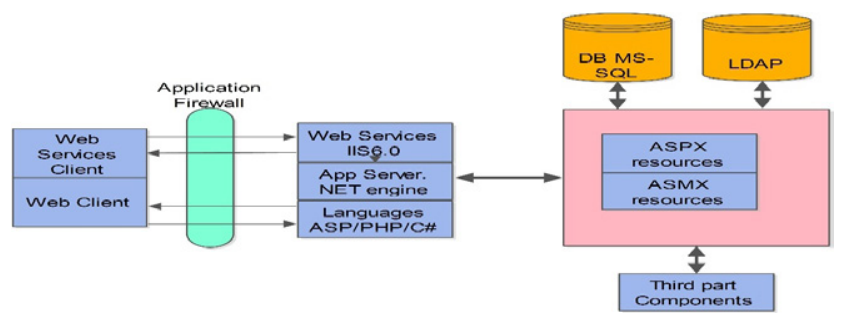

Figure 2. The Architecture of Web application

The web-based application has several dependencies[10]:

- A database: The web application has MS-SQL Server running as the backend database. This interface must be examined while performing a code review.

- $\quad$ The platform and web server. The application runs on the IIS web server with the .NET platform. This is helpful from two perspectives: 1) in securing deployment, and 2) in determining the source code type and language.

- Web resources and languages. In this paper, ASPX and ASMX are web resources. They are typical web applications and web services pages, written in the C\# language. These resources help to determine patterns during a code review.
- $\quad$ Authentication. The application authenticates users through an LDAP server. The authentication code is a critical component and requires analysis.

- $\quad$ Firewall. The application layer firewall is in place and content filtering must be enabled.

- Third-party components. Any third-party components being consumed by the application along with the integration code require analysis.

- Information access from the internet. Other aspects that require considerations are RSS feeds and emails, information that an application may consume from the internet.

\section{Embedded computer}

Computer system is part of a larger system and performs only peripheral functions; in this research Raspberry pi is use just for the communication between web application and robot controller.

\section{Robot control system}

This system has two main parts, which are the robotic arm and controller system. In this project, the Arduino Uno is the controller of the entire system. Arduino Uno will interface to the internet via an Arduino Ethernet shield, Arduino Ethernet shield will enable the Arduino Uno to interconnect to the internet via LAN cable[11]. Then, any computer or embedded computer that has internet connection can access and control the robotic arm. To enable the robotic arm for connection via the internet, relay infrastructure is used. Relay infrastructure enable secure Web access to embedded systems behind a firewall or NAT.

\section{Implementing}

The robot software complements the hardware architecture of the inspection robot by providing basic low-level hardware control that includes reading the input value from the internet and controlling the servo motors. Arduino Uno is a microcontroller board based on the ATmega328. It has 14 digital input/output pins (of which 6 can be used as PWM outputs), 6 analog inputs, a 16 $\mathrm{MHz}$ crystal oscillator, a USB connection, a power jack, an ICSP header, and a reset button. It contains everything needed to support the microcontroller. Arduino IDE: Arduino hardware is programmed by using a Wiringbased language (syntax and libraries), similar to $\mathrm{C}++$ with some slight simplifications and modifications, and a Processing-based integrated development environment. Raspberry pi is used as interface between web application and Arduino, at the Raspberry pi model B Linux operation system is used. All code for creating web application used Visual.Net, the Web application consist of 4 decision making for distributing parts. Motorola FX 7400 reader, Motorola AN200 antenna, and passive tags are used as RFID system in the implementation. Motorola FX 7400 reader consists of internet port for 
communication with web application based on the TCP/IP protocol. Figure 3 shows the activity diagram of the system started by arriving parts near to RFID gate (RFID antenna). RFID system read data and send to the web application via RFID reader, the web application makes decision and send real-time data control to the web client, robot control system receive data control via raspberry pi and Robot arm pick a part and put in the right location.

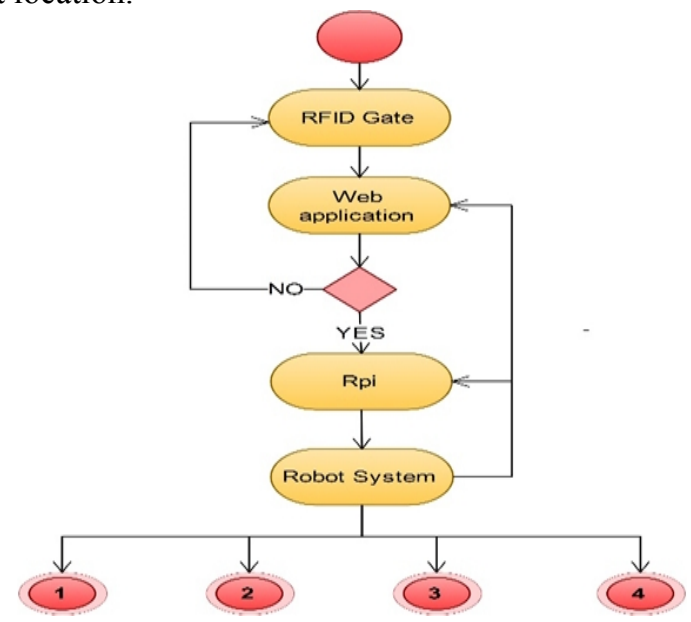

Figure 3. Activity diagram of system

Distributor Robot arm can be picked and place the parts with real-time control, and it does not depend on the sequence of part and can perform job with random mix. It has four different situations. Web based application with decoding information send it via RFID reader, enables Robot make a decision arrived arm and robot arm performs the job. Figure 4 show the implementation of the system.

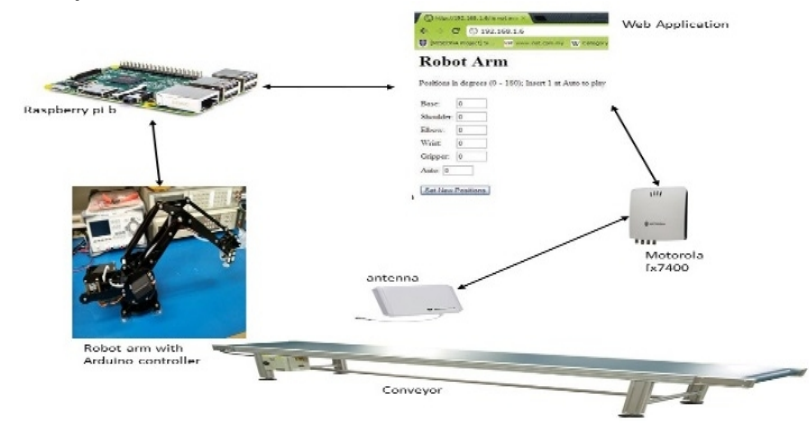

Figure 4. implementation of system

\section{Conclusion}

This paper proposes control system based on web-based application and RFID system for reconfigurable distributor robot arm in the random mix based parts. Using RFID sensor and web application not only increases the reconfiguration of the system but also increases the scalability of the control system by dividing hardware and software together. Proposed control system is implemented in the educational robot arm. The result of implantation will be discussed in the future work by considering real scenario.

\section{References}

1. A. V. Barenji, "An RFID-based distributed control system for flexible manufacturing system," Eastern Mediterranean University (EMU)-Doğu Akdeniz Üniversitesi (DAÜ), 2013.

2. A. Vatankhah Barenji, R. Vatankhah Barenji, and M. Hashemipour, "Structural modeling of a RFIDenabled reconfigurable architecture for a flexible manufacturing system," in Smart Objects, Systems and Technologies (SmartSysTech), Proceedings of 2013 European Conference on, 2013, pp. 1-10.

3. R. Vatankhah Barenji, M. Hashemipour, and D. A. Guerra-Zubiaga, "A framework for modelling enterprise competencies: from theory to practice in enterprise architecture," International Journal of Computer Integrated Manufacturing, pp. 1-20, 2014.

4. R. V. Barenji, A. V. Barenji, and M. Hashemipour, "A multi-agent RFID-enabled distributed control system for a flexible manufacturing shop," The International Journal of Advanced Manufacturing Technology, vol. 71, pp. 1773-1791, 2014.

5. S.-H. Suh, Y. Seo, S.-M. Lee, T.-H. Choi, G.-S. Jeong, and D.-Y. Kim, "Modelling and implementation of internet-based virtual machine tools," The International Journal of Advanced Manufacturing Technology, vol. 21, pp. 516-522, 2003.

6. X. Xu and S. T. Newman, "Making CNC machine tools more open, interoperable and intelligent-a review of the technologies," Computers in Industry, vol. 57, pp. 141-152, 2006.

7. L. Wang, P. Orban, A. Cunningham, and S. Lang, "Remote real-time CNC machining for web-based manufacturing," Robotics and Computer-Integrated Manufacturing, vol. 20, pp. 563-571, 2004.

8. R. Sepe Jr, "Remote web-based control," ed: Google Patents, 2004.

9. A. V. Barenji, R. V. Barenji, and M. Hashemipour, "A frameworks for structural modelling of an RFIDenabled intelligent distributed manufacturing control system," South African Journal of Industrial Engineering, vol. 25, pp. 48-66, 2014.

10. H.-W. Gellersen and M. Gaedke, "Object-oriented web application development," IEEE Internet Computing, vol. 3, pp. 60-68, 1999.

11. Y. Kintou, A. Nukuzuma, and S. Himeda, "Control system for controlling a mobile robot via communications line," ed: Google Patents, 2002. 